Article

\title{
Inhibition of osteopontin synthesis in THP-1 cells stimulated with phorbol 12-myristate 13-acetate by brefelamide derivatives
}

\author{
Gaowa Bai ${ }^{1^{+}}$, Takashi Matsuba ${ }^{2+}$, Haruhisa Kikuchi ${ }^{3}$, Hirotoshi Motoda ${ }^{1}$, Ryo Ozuru ${ }^{3}$, Haorile \\ Chagan-Yasutan $^{4}$, Osamu Yamada ${ }^{5}$, Yoshiteru Oshima ${ }^{3,6}$ and Toshio Hattori $^{*}$ \\ 1 Department of Health Science and Social Welfare, Kibi International University, Takahashi, Japan \\ 2 Division of Bacteriology, Department of Microbiology and Immunology, Faculty of Medicine, Tottori \\ University, Yonago, Tottori, Japan \\ 3 Laboratory of Natural Product Chemistry, Graduate School of Pharmaceutical Sciences, Tohoku \\ University, Sendai, Japan \\ 4 Mongolian Psychosomatic Medicine Department, International Mongolian Medicine Hospital of Inner \\ Mongolia, Hohhot, China \\ 5 Research and development center, FUSO Pharmaceutical Institute, LTD, Osaka, Japan \\ 6 Head Office for Open Innovation Strategy,Tohoku University, Sendai, Japan \\ * Correspondence: hattorit@kiui.ac.jp; Tel./Fax.: +81- 866-22-9469 \\ + These authors contributed equally to this work.
}

\begin{abstract}
Plasma osteopontin (OPN) levels are elevated in mycobacterium tuberculosis patients and may involve granuloma formation. New inhibitors using brefelamide, an aromatic amide isolated from Dictyostelium cellular slim molds which may inhibit OPN transcription at concentration of $1 \mu \mathrm{M}$, were synthesized as compounds $\mathrm{C}, \mathrm{D}$ and $\mathrm{E}$. Their inhibitory activity against OPN synthesis in phorbol 12-myristate 13-acetate (PMA)-stimulated THP-1 cells was confirmed using enzymelinked immunosorbent assay (ELISA), a multicolor immune-fluorescent microscope and western blot analysis. For the ELISA performed using the full-length OPN, each compound showed significant inhibition. Detailed analysis were done using $C$ and D. They also showed inhibitory activity when used on another ELISA system to detect the immune-related form of OPN and their IC50 were 0.6 and $1.2 \mu \mathrm{M}$ for compounds $\mathrm{C}$ and D, respectively. Fluorescent particle count of stained cell numbers by O-17 showed the inhibition. Antibodies for O-17 and 34E3, which recognize OPN N-terminus and thrombin-cleaved site, respectively, detected distinct bands on the western blots following PMA stimulation. The decrease in full-length OPN detected by O-17 in the compound-treated cells was identified via western blot analysis. These newly-developed compounds may therefore be used in clinical trials for cancer and infectious diseases.
\end{abstract}

Keywords: osteopontin; THP-1; brefelamide; inhibitor; tuberculosis.

1. Introduction 
Osteopontin (OPN), a calcium-binding glycophosphoprotein originally isolated from bone, mediates bone remodeling and tissue debridement [1]. OPN has been implicated in pathological and physiological processes such as cell proliferation and endothelial cell migration [2,3], and is expressed by macrophages. OPN also plays an important role in immunity, inflammation, tumor progression, cell viability [4,5], cell adhesion, proliferation, invasion, and apoptosis in tissue fibrosis [6], by binding to their receptors with integrin and CD44 variants [7]. Previously, we reported the upregulation of OPN in plasma of patients with adult $\mathrm{T}$ cell leukemia and dengue patients, implying its role in disease progression $[3,8]$.

OPN expression was also observed in the granuloma of mycobacterium tuberculosis (MTB) infected individuals [9], and a high level of plasma OPN was confirmed in subjects with MTB from the Philippines [10] and Indonesia [9]. Full-length OPN (FL-OPN), its intact form, serves as a protease(s) cleavage target. During this process, fragments of OPN are produced. Among these fragments, proteolytic cleavage of FL-OPN by thrombin (between Arg168 and Ser169) generates the functional fragment, N-terminal thrombin-cleaved OPN (tr-OPN), which contains cryptic binding sites for the integrins, $\alpha 9 \beta 1$ and $\alpha 4 \beta 1$, enhancing tr-OPN attachment to integrin. [11] Increases in trOPN levels have been reported in the recovery phase of dengue virus (DENV) infection. Furthermore, another OPN form, undefined OPN (Ud-OPN), was detected in DENV infections when a different ELISA system is employed [8].

Higher plasma concentrations of Ud-OPN, but not FL-OPN or tr-OPN, positively correlates with neutrophil numbers and negatively correlates with TB-specific memory T-cell numbers, and Ud-OPN is dependent on PMA-stimulation, indicating Ud-OPN represenet immune-relared form of OPN [12].

It is also known that other enzymes such as matrix metalloproteinases (MMPs) can cleave OPN at non-thrombin cleavage sites [13,14]. Accumulation of $\alpha 4 \beta 1$ and other integrin-bearing cells is reported in MTB infection [15]. Recently, we showed that small amounts of FL-OPN and tr-OPN were detected in the supernatants of non- phorbol 12-myristate 13-acetate (PMA)-stimulated cells by ELISA, with increased levels of all forms, including undefined Ud-OPN, in PMA-stimulated cells [16]. Brefelamide has been reported to inhibit OPN expression in A549 cells at a concentration of 50 $\mu \mathrm{M}$ using an OPN promoter reporter assay [17]. We also reported that brefelamide and its methyl ether derivative (compound B; Figure. 1) suppressed OPN production in DENV-infected THP-1 cells and also inhibited DENV replication [18]. Recently, we developed three novel derivatives which exhibited inhibitory activity at lower concentrations and examined their effect on OPN production in PMA-stimulated THP-1 cells.

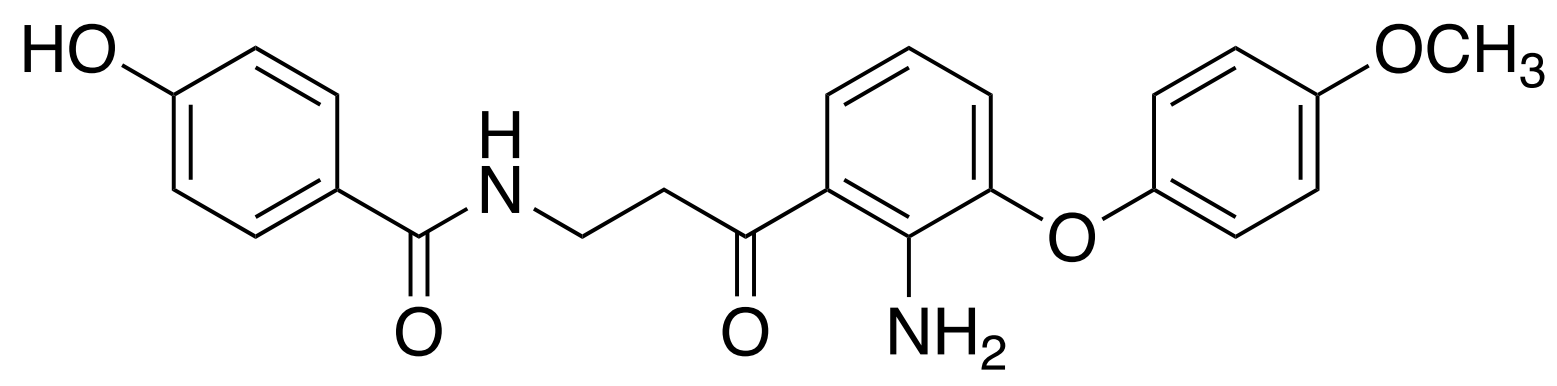

Figure 1. Structure of compound B. 


\section{Results}

\subsection{Inhibition of OPN expression by brefelamide}

The structure of compound B has already been elucidated (Figure.1) [18], while those of the other new compounds (C, D, and E) will be described elsewhere (unpublished data). Luciferase expression in A549/OPN-luc cells was dose-dependently suppressed by the compounds; compound B showed the highest IC50 when compared to the other compounds which showed lower IC50 values (Table 1).

Table1. The compounds and their ability to inhibit transcriptional activity

\begin{tabular}{cc}
\hline Compound & OPNluc IC50 $(\mu \mathrm{M})$ \\
\hline B & 10.2 \\
C & 1.0 \\
D & 1.1 \\
E & 1.2 \\
\hline
\end{tabular}

[A1]

\subsection{ELISA}

OPN from the cell supernatant cultured with PMA and the newly-developed compounds were measured by ELISA; the compounds were added at three concentrations $(5,2.5,1.25 \mu \mathrm{M})$ (Figure 4). In the ELISA system which measured FL-OPN, B did not exert inhibitory activity; C showed dosedependent inhibitory activity; and $\mathrm{E}$ and D also suppressed the synthesis of FL-OPN. The IC50 values for C, D, and E were 2.6, 2.3 and 3.1 $\mu \mathrm{M}$, respectively. As compounds C and D showed the lower IC50 values, we examined and compared the effect of compounds C and D on FL-OPN and Ud-OPN. In this experiment thereafter, pretreatment with the compounds, $2 \mathrm{~h}$ before PMA addition, was performed for the analysis. The IC50 values of compounds C and D were 1.6 and $1.8 \mu \mathrm{M}$, respectively, for Fl-OPN, indicating that pretreatment was more effective. The compounds also showed inhibitory activity in the Ud-OPN assay as their IC50 values were 0.6 and $1.2 \mu \mathrm{M}$ for compounds $\mathrm{C}$ and $\mathrm{D}$, respectively. 
(a)

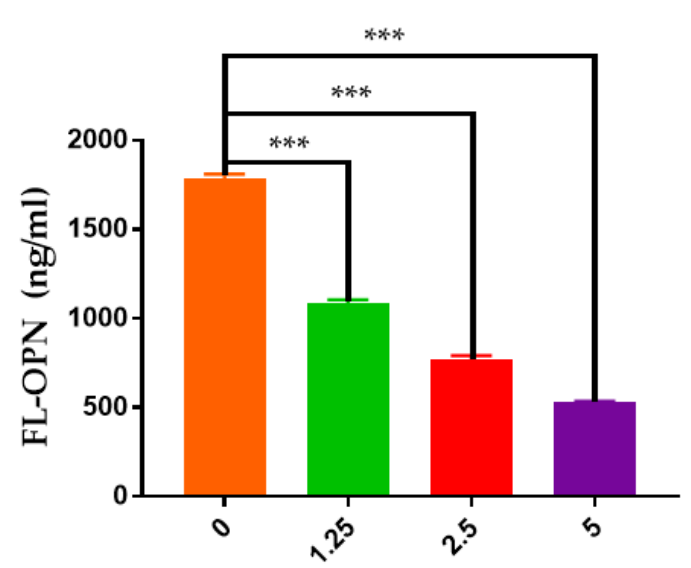

Compound $\mathrm{C}(\mu \mathrm{M})$
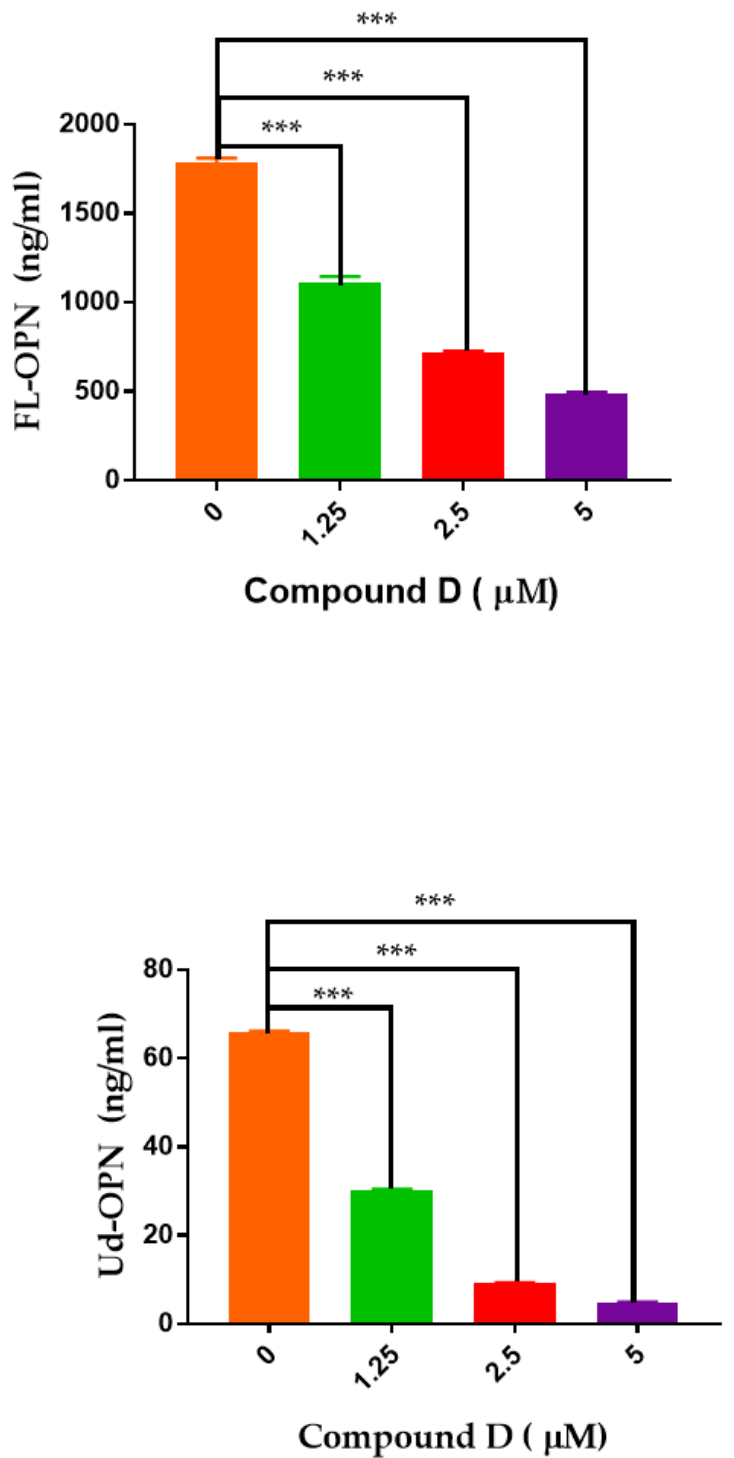

Figure 2. Effect of compounds $C$ and D on OPN synthesis. (a) FL-OPN, (b) Ud-OPN were measured. ${ }^{* * *} P<0.001$, Multiple $t$ test performed by prism software.

\subsection{Immunofluorescence study}

Multicolor immune-fluorescent analysis of OPN was conducted using the compound-treated PMAstimulated THP-1 cells. The characterization of each antibody in PMA-stimulated THP-1 cells was previously described [16]. Polyclonal rabbit antibody, O-17, specific to the N-terminus of OPN (Ile17Gln31) and a mouse monoclonal antibody34E3, specific to the epitope Ser162-Arg168, which is exposed by thrombin digestion were used. [11] In this experiment, pretreatment with the compounds C and D $5 \mu \mathrm{M}, 2 \mathrm{~h}$ before PMA addition, was performed for the analysis. (Figure 3). Compounds $\mathrm{C}$ and D apparently suppressed cytoplasmic staining of O-17. 


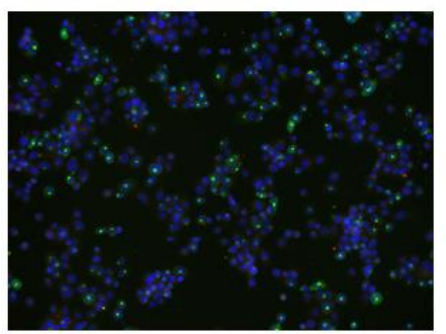

Control

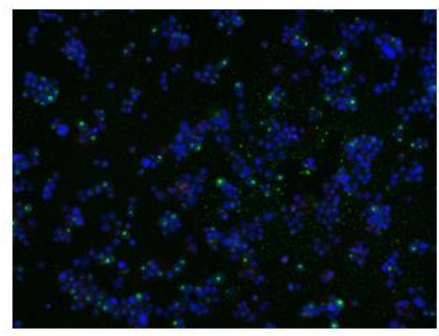

Compound C

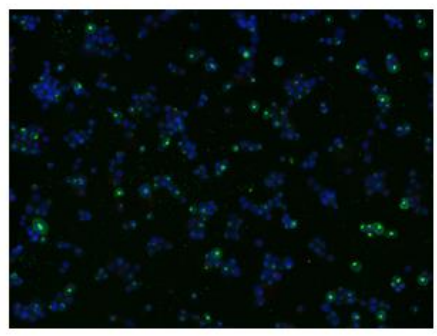

Compound D

Figure 3. Two color analysis of compound-treated PMA-stimulated THP-1 cells. O-17(AF488) and 34E3(AF546) were used (x200) Immuno-fluorescence was numerically analyzed by the Fiji. OPN staining was decreased to almost half by compounds C and D treatment. A few percentages of cells were positive against 34E3 staining in control cells, no positive staining cells were enumerated in compounds treated cells. (Figure 4).

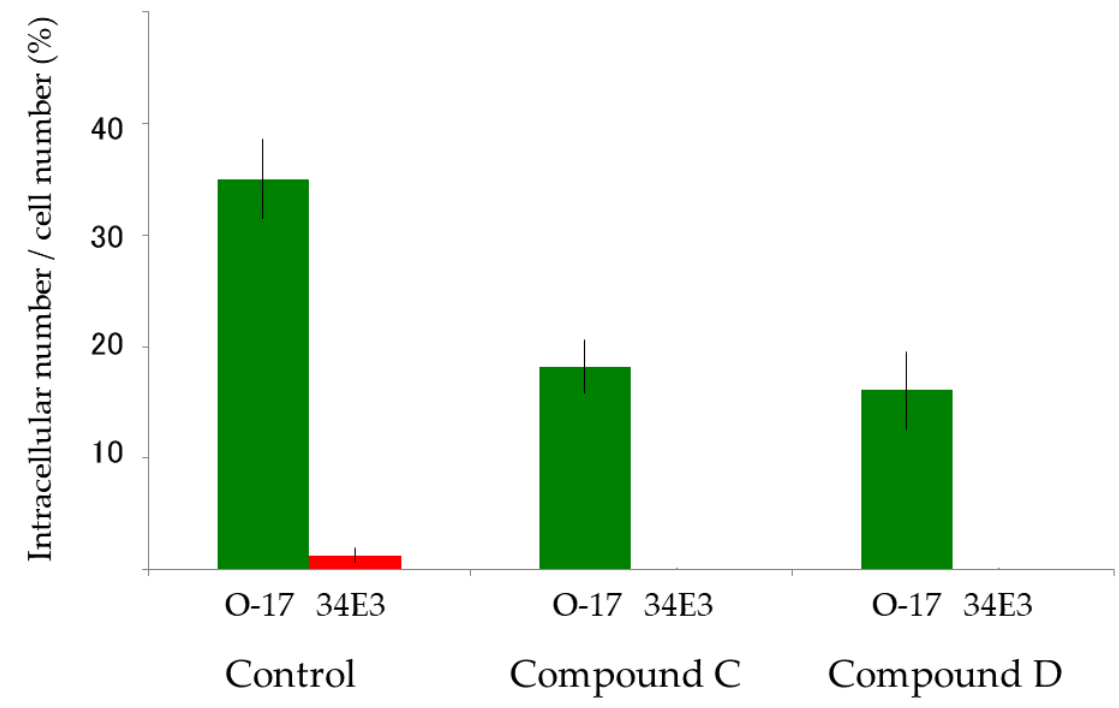

Figure 4. The numerical representation of fluorescence in the particle analysis of Fijii. Fluorescence obtained with: Left, O-17; Right, 34E3.

\subsection{Western blot}

To confirm the effect of these compounds OPN protein expression, we utilized two antibodies that identify different epitopes of OPN. In this experiment, pretreatment with the compounds C and D $5 \mu \mathrm{M}, 2 \mathrm{~h}$ before PMA addition, was performed for the analysis. A definitive band was observed in PMA treated cells, while these bands were not seen in medium cultured cells, while comparable amounts of proteins were loaded as shown in anti-actin antibody (Figure 5). FL-OPN (70-kD band) and smaller fragments were detected by O-17. The apparent reduction of the bands corresponding to full length OPN these bands was observed in the compound-treated PMA-THP-1 cells. 34E3 
monoclonal antibody identified two discrete bands as indicated by arrow (33kDa) and asterisks as described before. [16] Slight reduction of 33kDa band were seen in compound treated cells.

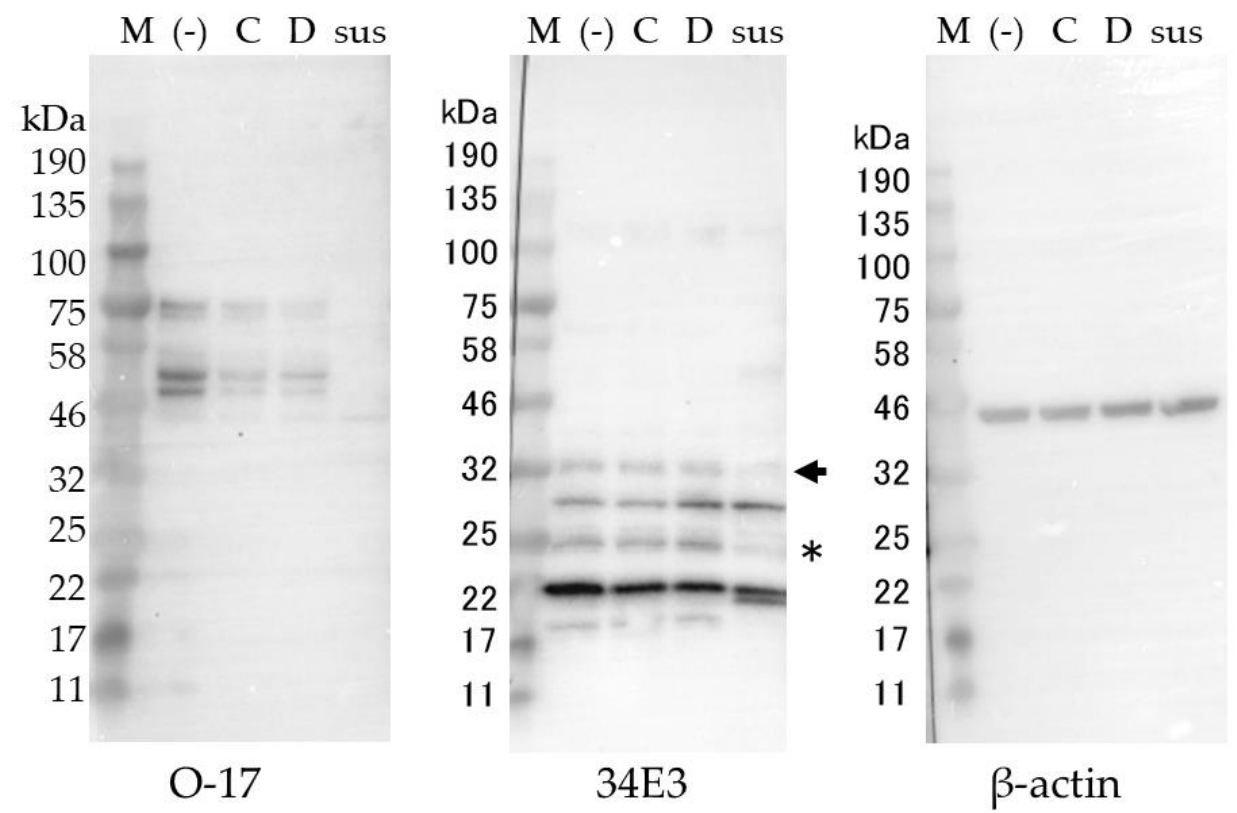

Figure 5. Western blot analysis of lysates from PMA-stimulated THP-1 cells cultured with the indicated compounds; the O-17 antibody, 34E3 and anti- $\beta$-actin antibody were used. Cells were cultured with $30 \mathrm{ng} / \mathrm{mL}$ of PMA for 2 days with the compounds (C,D, $5 \mu \mathrm{M})$, and the cell lysates were subjected to SDS-PAGE (5-20\%) followed by immunostaining. (-), PMA only; C,D, compounds C,D; sus, cells cultured medium only.

\section{Discussion}

In this study, we confirmed that the new compounds generated with brefelamide, a compound reported to inhibit OPN expression and protein synthesis at $50 \mu \mathrm{M}$, inhibited at lower concentrations (less than $1 \mu \mathrm{M}$ ) than previously observed. Brefelamide is known to induce Smad 4, which is associated with TGF- $\beta$ signaling, followed by inhibiting OPN synthesis [17]. OPN may also be cleaved in THP-1 cells upon BCG infection or PMA stimulation [16]. To analyze the metabolism of OPN products in THP-1 cells, we developed 3 new compounds (C, D, and E). The chemical structure of compound B was previously reported [18]; B had the highest IC50 when compared to C-E based on the transcription assay performed (Table1). In ELISA for FL-OPN, similarly C-E shows lower IC50 values. Unexpectedly, more inhibition on Ud-OPN ELISA than those on FL-OPN ELISA were observed. Using the antibody of the N-terminus of OPN, O-17, evident suppression of OPN synthesis in the compound-treated THP-1 cells was observed by the immuno-fluorescent assay and western blot analysis. In immuno-fluorescent study, the low expression of 34E3 signal, which is specific to the thrombin-cleaved site, SVVYGLR, was observed but were not detected in compounds treatment. This epitope was deemed a thrombin cleavage site and was also found in PMA-stimulated THP-1 cells as previously reported [16]. Cleaved and/or modified products exhibit greater biological activities by binding to different proteins in the integrin family [11]. In previous studies using patients with pulmonary TB, Ud-OPN had a negative correlation with the number of ESAT-6 specific IFN- $\gamma$ spot-forming cells, and a positive correlation with neutrophil numbers; FL-OPN or tr-OPN did not show such correlations [12]. These findings indicate that Ud-OPN may possess more immune-related functions than FL-OPN and tr-OPN; the molecular size of OPN using the Ud-OPN ELISA is unknown. 
In a previous experiment, Ud-OPN levels were found to decrease following the removal of PMA from the culture, while synthesis of full-length OPN and tr-OPN continued; this indicates Ud-OPN synthesis is PMA-dependent. [16] In PMA stimulation, cells express proteases [19], degradation of OPN may be facilitated but how these molecules participate in Ud-OPN synthesis were not known. It should also be noted that the immune check point molecule, PD-L1, is dependent on OPN; therefore, OPN suppression may affect other molecules, which could promote the degradation of OPN [20]. More detailed analysis of OPN metabolism in PMA-stimulated THP-1 cells is required to clarify the metabolism of OPN.

Other researchers, and us, have reported elevated OPN levels and its association with the severity of MTB infection [9,10] [12] [21]. OPN was also claimed to serve as a reliable prognostic indicator of improvement during the early treatment of pulmonary TB [22]. On the contrary, it was suggested that the level of OPN protein expression correlates with effective immune and inflammatory responses [23].

Immuno-histochemical analysis has demonstrated its presence in granuloma and its association with macrophages; the levels of Ud-OPN were positively associated with neutrophil numbers and negatively associated with lymphocyte numbers [12]. Increased osteopontin expression was also found to be associated with silicosis, another granulomatous disease. Therefore, osteopontin may participate in granuloma formation [24].

We have clearly demonstrated that OPN synthesis is effectively inhibited in vitro at less than 1 $\mu \mathrm{M}$ by the newly-developed compounds. The behavior of these compounds in MTB infection in vivo would therefore serve as an interesting future project. The therapy for TB is hampered by many factors and granuloma formation may function as one of the major causes of poor drug delivery, causing drug resistance. The development of adjunctive, granuloma-targeted therapy, similar to other host-directed therapies, may be beneficial in increasing the availability of approved drugs to aid in the treatment and prevention of TB. [25]Neutrophil numbers which are associated with OPN levels, are known risk factors of TB. [26]

Statins, which suppress OPN production [27], and their use has been associated with a reduced risk of developing active TB in some studies [28]. Application of the newly-generated compounds to host-directed therapy may be possible.

In conclusion, we have reported that novel brefelamide-derived compounds suppressed fulllength OPN production in PMA-stimulated THP-1 cells. These compounds also suppressed UD-OPN which may serve as the immune-related form of OPN.

\section{Materials and Methods}

\subsection{Chemicals}

Brefelamide is an aromatic amide isolated from methanol extracts of Dictyostelium brefeldianum and D. giganteum slime mold fruiting bodies [29]; its derivative is referred to as compound B, which contains a methyl ether group (-OCH3) (Figure 1) [18]. Compounds C, D and E were also synthesized (unpublished data). The compounds were dissolved in DMSO at a concentration of $50 \mathrm{mmol} / \mathrm{l}$ and stored at $-20^{\circ} \mathrm{C}$. Aliquots of the stock solutions were subsequently diluted to the indicated concentrations before use to treat the cells. 


\subsection{Cell lines and culture}

A549 cells and Human THP-1 cells derived from acute monocytic leukemia patients were obtained from the American Type Culture Collection (Manassas, VA, USA). Cells were maintained in Dulbecco's modified Eagle's medium (DMEM; Invitrogen) or Roswell Park Memorial Institute (RPMI) 1640 medium (Wako Pure Chemical Industries Ltd, Osaka, Japan), in the presence of 10\% fetal bovine serum (FBS; Thermo Fisher Scientific, Waltham, MA, USA), 50U/ml penicillin and streptomycin in a $5 \% \mathrm{CO}_{2}$ humidified atmosphere. PMA (Wako Pure Chemical Industries, Osaka, Japan) $(30 \mathrm{ng} / \mathrm{ml})$ was used to induce OPN expression.

\subsection{Plasmids Transient transfection and luciferase assay}

The reporter vector, pOPN1-luc, has been previously described [17]. A549 cells were seeded in 12 -well plates at $1 \times 10^{5} \mathrm{in} 1 \mathrm{ml}$ medium/well, $24 \mathrm{~h}$ before transfection. The indicated plasmid DNA was transfected into cells with Effectene Transfection Reagent (Qiagen). For each transient transfection, pRL-TK vector (Promega) was co-transfected as an internal control to normalize transfection efficiency. Cells were harvested at 48-h post-transfection, and the cell lysates prepared for the luciferase assay with Dual-Luciferase Reporter Assay System (Promega) according to the manufacturer's instructions. Luciferase activity was measured using a GloMax ${ }^{\circledR}$ 20/20 Luminometer (Promega). Cell viabilities were tested using cell proliferation reagent (Roche diagnostic) according to the manufactures instructions. The half maximal inhibitory concentration (IC50) of compounds was determined from the dose-response curve as described before [17]

\subsection{Effect of compounds on PMA-stimulated THP-1 cells}

THP- 1 cells were seeded in 96-well tissue culture plates at a density of $1.8 \times 10^{4} /$ well or $10 \mathrm{ml}$ flasks for $24 \mathrm{~h}$ at $37^{\circ} \mathrm{C}$ in a humidified $5 \% \mathrm{CO}_{2}$ atmosphere. The following day, $10 \mu \mathrm{l}$ of the compounds at concentrations ranging from 1 to $25 \mu \mathrm{M}$ were added at the same time or $2 \mathrm{~h}$ before the addition of PMA (30 ng/ml). After incubation for $48 \mathrm{~h}$, culture supernatants were obtained for ELISA, and the cells obtained for immuno-fluorescent or western blot analysis following centrifugation $(4,000 \mathrm{rpm}$, $3 \mathrm{~min}$ ) as previously described [15].

\subsection{Enzyme-linked immunosorbent assay (ELISA)}

To identify FL-OPN, an ELISA kit (IBL, Gunma, Japan) was used [8]. In the FL-OPN kit, O-17, a polyclonal rabbit antibody specific to the N-terminus of OPN was used as a capture antibody, and the mouse monoclonal antibody, 10A16, served as the detector antibody.

Ud-OPN in culture supernatants were determined using the Human Osteopontin DuoSet ELISA Development System Kit (R\&D Systems, Minneapolis, MN, USA) [3]. The proprietary capture monoclonal antibody and the detection polyclonal antibodies in this ELISA kit were both generated against recombinant human OPN (NS0-derived; amino acids, Ile17-Asn300); the epitopes for these 
antibodies were not disclosed. The amount of the synthesized OPN was calculated based on viable cell numbers, which were determined with the Cell Titer 96 Aqueous Non-Radioactive Cell Proliferation System (Promega). Cells were seeded in 96-well tissue culture plates at a density of $2 \times$ $10^{4} /$ well in $50 \mu$ of complete medium followed by the addition of compounds and PMA) to each well. After incubation for $48 \mathrm{~h}, 20 \mu \mathrm{l}$ per well of 3-(4,5-dimethyl-2-yl)-5-(3-carboxymethoxyphenyl)2-(4sulfophenyl)-2H-tetrazolium salt/phenazine methosulfate solution was added to each well, followed by incubation for $4 \mathrm{~h}$. The absorbance at $492 \mathrm{~nm}$ was recorded with an ELISA plate reader.. The IC50 value was determined by identifying the $\mathrm{X}$-axis value corresponding to $\mathrm{N}$-half of the difference between the maximum and minimum absorbance values using GraphPad Prism 7 software (GraphPadInc., San Diego, CA, USA) as described . [18]

\subsection{Immunofluorescence}

PMA stimulated THP-1 cells cultured with compounds on 2-well chambered glass slides (Nunc Lab-Tek ${ }^{\mathrm{TM}}$,Thermo Fisher Scientific, MA, USA) were fixed with $4 \%$ (w/v) paraformaldehyde in PBS (-) for $20 \mathrm{~min}$ at room temperature. The cells were incubated for $1 \mathrm{~h}$ with PBS (-) containing $0.1 \%(\mathrm{v} / \mathrm{v})$ Triton-X100 for $5 \mathrm{~m}$ at room temperature, $1 \%(\mathrm{v} / \mathrm{v})$ bovine serum albumin (BSA) and each of the appropriate primary antibodies including O-17 (1:400 dilution; IBL, Gunma, Japan), 34E3 (1:400 dilution; IBL), and 10A16 (1:400 dilution; IBL). The secondary antibodies goat antirabbit IgG $(\mathrm{H}+\mathrm{L})$ - Alexa Fluor ${ }^{\circledR} \mathrm{AF} 488$ (AF488) and goat anti-mouse IgG (H+L)-Alexa Fluor ${ }^{\circledR} 546$ (AF546) were purchased from Thermo Fisher Scientific. For nuclear staining, 4', 6-diamidino-2phenylindole (DAPI) was used. Each of the primary antibodies, the secondary antibodies, and DAPI were diluted to $0.5 \mu \mathrm{g} / \mathrm{ml}, 5 \mu \mathrm{g} / \mathrm{ml}$ and $1 \mu \mathrm{g} / \mathrm{ml}$ with PBS(-) containing $1 \%(\mathrm{w} / \mathrm{v})$ BSA, respectively and incubated each for $1 \mathrm{~h}$ at room temperature. Between incubations, cells were washed twice with PBS (-). The cover glass was applied onto glass slides and the images captured with a BZ-X700 fluorescence microscope (Keyence, Osaka, Japan). Quantification was performed using the Fiji.[30] The signals of DAPI as numbers of cells and the signals of O-17 or 34E3 were counted. The fluorescent images were divided into each RGB channels, and these were converted into 16-bit grayscale images. The signals of blue channel (DAPI) were selected with auto-threshold and counted by the menu command of Analyze Particle. The intracellular signals of green (O-17) or red (34E3) channels were selected with manual threshold and counted in the same way.

\subsection{Western blot}

Washed THP-1 cells $\left(1.5 \times 10^{6}\right)$ were lysed on ice for 15 min using $300 \mu$ lof lysis buffer provided in the WSE-7420 EzRIPA Lysis kit (ATTO Corporation, Tokyo, Japan). Supernatants were then processed by centrifugation at $14,000 \times g$ for $15 \mathrm{~min}$, according to the manufacturer's protocol. Protein concentration was then determined by Bradford Protein Assay kit (Takara Bio Inc., Shiga, Japan). Six $\mu \mathrm{g}$ from each sample was separated by sodium dodecyl sulfate-polyacrylamide gel electrophoresis (SDS-PAGE) using 5-20\% gradient gels, followed by western blot analysis using semi-dry transfer apparatus (Bio Craft, Tokyo, Japan). Antibody was diluted using Western BLoT Immuno Booster solutions (Takara Bio). O-17 antibody (0.1 $\mu \mathrm{g} / \mathrm{mL}$; IBL, Gunma, Japan), 34E3 $0.1 \mu \mathrm{g} / \mathrm{mL}$ dilution; IBL) and anti- $\beta$ actin (1:20,000 dilution; A5316, Sigma-Aldrich Co. LLC, St. Louis, USA) were used as the 
primary antibody. Bound primary antibody was reacted with horseradish peroxidase-conjugated goat anti-rabbit IgG or anti-mouse IgG (1:200,000 dilution;Sigma-Aldrich Co. LLC, St. Louis, USA), and visualized using a Immobilon ${ }^{\circledR}$ Forte Western membrane Substrate (Merck KGaA, Darmstadt, Germany) according to the manufacturer's protocol.

\subsection{Statistical analysis}

Unpaired $\mathrm{t}$ tests (two tailed) were used to compare the results obtained from the BCG infected and mock-infected cells. $\mathrm{P}$ values $<0.05$ were considered statistically significant. All statistical analyses were performed using GraphPad Prism software, version 6 (GraphPad Software Inc., San Diego, CA, USA).

\section{Conclusions}

Novel brefelamide-derived compounds suppressed the transcription of OPN, FL-OPN and UdOPN synthesis in PMA-treated THP-1 cells at a concentration less than $1 \mu \mathrm{M}$. As Ud-OPN includes immune-related OPN, the new compounds could be used as a host-directed therapy against MTB infection.

Author Contributions: methodology, Y.O. and H.K.; investigation, O.Y., G.B., R.O. and T.M.; formal analysis, O.Y., G.B., R.O. and T.M; data curation, H.C-Y.; conceptualization, T.M., O.Y. and T.H.; writing, T.M., O.Y. and T.H.

Funding: This research was funded by the Research Program on Emerging and Re-Emerging Infectious Diseases from the Japan Agency for Medical Research and Development (AMED) 18fk0108042h0002, and partially supported by the Japan Society for the Promotion of Science (JSPS) Grants-in-Aid for Scientific Research (KAKENHI), Grant Number JP17H01690.

Acknowledgments: We are grateful for Junsei Educational Institute for support.

Conflicts of Interest: The authors declare no conflict of interest.

\begin{tabular}{ll}
\multicolumn{2}{l}{ Abbreviations } \\
OPN & Osteopontin \\
FL-OPN & Full-length OP \\
Tr-OPN & Thrombin cleaved form of OPN \\
Ud-OPN & Undefined OPN \\
PMA & Phorbol 12-myristate 13-acetate
\end{tabular}

References

1. Giachelli, C.M.; Steitz, S. Osteopontin: a versatile regulator of inflammation and biomineralization. Matrix Biol 2000, 19, 615-622. 
2. Senger, D.R.; Ledbetter, S.R.; Claffey, K.P.; Papadopoulos-Sergiou, A.; Peruzzi, C.A.; Detmar, M. Stimulation of endothelial cell migration by vascular permeability factor/vascular endothelial growth factor through cooperative mechanisms involving the alphavbeta3 integrin, osteopontin, and thrombin. Am J Pathol 1996, 149, 293 305.

3. Chagan-Yasutan, H.; Tsukasaki, K.; Takahashi, Y.; Oguma, S.; Harigae, H.; Ishii, N.; Zhang, J.; Fukumoto, M.; Hattori, T. Involvement of osteopontin and its signaling molecule CD44 in clinicopathological features of adult T cell leukemia. Leuk Res 2011, 35, 1484-1490, doi:10.1016/j.leukres.2011.05.011.

4. Mazzali, M.; Kipari, T.; Ophascharoensuk, V.; Wesson, J.A.; Johnson, R.; Hughes, J. Osteopontin--a molecule for all seasons. QJM 2002, 95, 3-13.

5. El-Tanani, M.K.; Campbell, F.C.; Kurisetty, V.; Jin, D.; McCann, M.; Rudland, P.S. The regulation and role of osteopontin in malignant transformation and cancer. Cytokine Growth Factor Rev 2006, 17, 463-474, doi:10.1016/j.cytogfr.2006.09.010.

6. Kato, A.; Okura, T.; Hamada, C.; Miyoshi, S.; Katayama, H.; Higaki, J.; Ito, R. Cell stress induces upregulation of osteopontin via the ERK pathway in type II alveolar epithelial cells. PLoS One 2014, 9, e100106, doi:10.1371/journal.pone.0100106.

7. Murphy-Ullrich, J.E.; Sage, E.H. Revisiting the matricellular concept. Matrix Biol 2014, 37, 1-14, doi:10.1016/j.matbio.2014.07.005.

8. Chagan-Yasutan, H.; Lacuesta, T.L.; Ndhlovu, L.C.; Oguma, S.; Leano, P.S.; Telan, E.F.; Kubo, T.; Morita, K.; Uede, T.; Dimaano, E.M., et al. Elevated levels of fulllength and thrombin-cleaved osteopontin during acute dengue virus infection are associated with coagulation abnormalities. Thromb Res 2014, 134, 449-454, doi:10.1016/j.thromres.2014.05.003.

9. Hasibuan, F.M.; Shiratori, B.; Senoputra, M.A.; Chagan-Yasutan, H.; Koesoemadinata, R.C.; Apriani, L.; Takahashi, Y.; Niki, T.; Alisjahbana, B.; Hattori, T. Evaluation of matricellular proteins in systemic and local immune response to Mycobacterium tuberculosis infection. Microbiol Immunol 2015, 59, 623-632, doi:10.1111/1348-0421.12320.

10. Shiratori, B.; Leano, S.; Nakajima, C.; Chagan-Yasutan, H.; Niki, T.; Ashino, Y.; Suzuki, Y.; Telan, E.; Hattori, T. Elevated OPN, IP-10, and neutrophilia in loopmediated isothermal amplification confirmed tuberculosis patients. Mediators Inflamm 2014, 2014, 513263, doi:10.1155/2014/513263.

11. Kon, S.; Yokosaki, Y.; Maeda, M.; Segawa, T.; Horikoshi, Y.; Tsukagoshi, H.; Rashid, M.M.; Morimoto, J.; Inobe, M.; Shijubo, N., et al. Mapping of functional epitopes of osteopontin by monoclonal antibodies raised against defined internal sequences. J Cell Biochem 2002, 84, 420-432.

12. Shiratori, B.; Zhao, J.; Okumura, M.; Chagan-Yasutan, H.; Yanai, H.; Mizuno, K.; Yoshiyama, T.; Idei, T.; Ashino, Y.; Nakajima, C., et al. Immunological Roles of Elevated Plasma Levels of Matricellular Proteins in Japanese Patients with Pulmonary Tuberculosis. Int J Mol Sci 2016, 18, doi:10.3390/ijms18010019.

13. Christensen, B.; Schack, L.; Klaning, E.; Sorensen, E.S. Osteopontin is cleaved at multiple sites close to its integrin-binding motifs in milk and is a novel substrate for 
plasmin and cathepsin D. J Biol Chem 2010, 285, 7929-7937, doi:10.1074/jbc.M109.075010.

14. Agnihotri, R.; Crawford, H.C.; Haro, H.; Matrisian, L.M.; Havrda, M.C.; Liaw, L. Osteopontin, a novel substrate for matrix metalloproteinase-3 (stromelysin-1) and matrix metalloproteinase-7 (matrilysin). J Biol Chem 2001, 276, 28261-28267, doi:10.1074/jbc.M103608200.

15. Mattila, J.T.; Beaino, W.; Maiello, P.; Coleman, M.T.; White, A.G.; Scanga, C.A.; Flynn, J.L.; Anderson, C.J. Positron Emission Tomography Imaging of Macaques with Tuberculosis Identifies Temporal Changes in Granuloma Glucose Metabolism and Integrin alpha4beta1-Expressing Immune Cells. Journal of immunology 2017, 199, 806-815, doi:10.4049/jimmunol.1700231.

16. Bai, G.; Motoda, H.; Ozuru, R.; Chagan-Yasutan, H.; Hattori, T.; Matsuba, T. Synthesis of a Cleaved Form of Osteopontin by THP-1 Cells and Its Alteration by Phorbol 12-Myristate 13-Acetate and BCG Infection. Int J Mol Sci 2018, 19, doi:10.3390/ijms19020418.

17. Zhang, J.; Yamada, O.; Kida, S.; Matsushita, Y.; Murase, S.; Hattori, T.; Kubohara, Y.; Kikuchi, H.; Oshima, Y. Identification of brefelamide as a novel inhibitor of osteopontin that suppresses invasion of A549 lung cancer cells. Oncol Rep 2016, 36, 2357-2364, doi:10.3892/or.2016.5006.

18. Pascapurnama, D.N.; Labayo, H.K.; Dapat, I.; Nagarajegowda, D.D.; Zhao, J.; Zhang, J.; Yamada, O.; Kikuchi, H.; Egawa, S.; Oshima, Y., et al. Induction of Osteopontin by Dengue Virus-3 Infection in THP-1 Cells: Inhibition of the Synthesis by Brefelamide and Its Derivative. Front Microbiol 2017, 8, 521, doi:10.3389/fmicb.2017.00521.

19. Kang, J.H.; Kim, J.K.; Park, W.H.; Park, K.K.; Lee, T.S.; Magae, J.; Nakajima, H.; Kim, C.H.; Chang, Y.C. Ascochlorin suppresses oxLDL-induced MMP-9 expression by inhibiting the MEK/ERK signaling pathway in human THP-1 macrophages. J Cell Biochem 2007, 102, 506-514, doi:10.1002/jcb.21312.

20. Zhang, Y.; Du, W.; Chen, Z.; Xiang, C. Upregulation of PD-L1 by SPP1 mediates macrophage polarization and facilitates immune escape in lung adenocarcinoma. Exp Cell Res 2017, 359, 449-457, doi:10.1016/j.yexcr.2017.08.028.

21. Koguchi, Y.; Kawakami, K.; Uezu, K.; Fukushima, K.; Kon, S.; Maeda, M.; Nakamoto, A.; Owan, I.; Kuba, M.; Kudeken, N., et al. High plasma osteopontin level and its relationship with interleukin-12-mediated type $1 \mathrm{~T}$ helper cell response in tuberculosis. Am J Respir Crit Care Med 2003, 167, 1355-1359, doi:10.1164/rccm.200209-1113OC.

22. Zhu, Y.; Jia, H.; Chen, J.; Cui, G.; Gao, H.; Wei, Y.; Lu, C.; Wang, L.; Uede, T.; Diao, H. Decreased Osteopontin Expression as a Reliable Prognostic Indicator of Improvement in Pulmonary Tuberculosis: Impact of the Level of Interferon-gammaInducible Protein 10. Cell Physiol Biochem 2015, 37, 1983-1996, doi:10.1159/000438559.

23. Nau, G.J.; Chupp, G.L.; Emile, J.F.; Jouanguy, E.; Berman, J.S.; Casanova, J.L.; Young, R.A. Osteopontin expression correlates with clinical outcome in patients with 
mycobacterial infection. Am J Pathol 2000, 157, 37-42, doi:10.1016/S00029440(10)64514-2.

24. Nau, G.J.; Guilfoile, P.; Chupp, G.L.; Berman, J.S.; Kim, S.J.; Kornfeld, H.; Young, R.A. A chemoattractant cytokine associated with granulomas in tuberculosis and silicosis. Proc Natl Acad Sci U S A 1997, 94, 6414-6419.

25. Stek, C.; Allwood, B.; Walker, N.F.; Wilkinson, R.J.; Lynen, L.; Meintjes, G. The Immune Mechanisms of Lung Parenchymal Damage in Tuberculosis and the Role of Host-Directed Therapy. Front Microbiol 2018, 9, 2603, doi:10.3389/fmicb.2018.02603.

26. Kiran, D.; Podell, B.K.; Chambers, M.; Basaraba, R.J. Host-directed therapy targeting the Mycobacterium tuberculosis granuloma: a review. Semin Immunopathol 2016, 38, 167-183, doi:10.1007/s00281-015-0537-x.

27. Matsuura, M.; Suzuki, T.; Suzuki, M.; Tanaka, R.; Ito, E.; Saito, T. Statin-mediated reduction of osteopontin expression induces apoptosis and cell growth arrest in ovarian clear cell carcinoma. Oncol Rep 2011, 25, 41-47.

28. Lai, C.C.; Lee, M.T.; Lee, S.H.; Hsu, W.T.; Chang, S.S.; Chen, S.C.; Lee, C.C. Statin treatment is associated with a decreased risk of active tuberculosis: an analysis of a nationally representative cohort. Thorax 2016, 71, 646-651, doi:10.1136/thoraxjnl2015-207052.

29. Kikuchi, H.; Saito, Y.; Sekiya, J.; Okano, Y.; Saito, M.; Nakahata, N.; Kubohara, Y.; Oshima, Y. Isolation and synthesis of a new aromatic compound, brefelamide, from dictyostelium cellular slime molds and its inhibitory effect on the proliferation of astrocytoma cells. J Org Chem 2005, 70, 8854-8858, doi:10.1021/jo051352x.

30. Schindelin, J.; Arganda-Carreras, I.; Frise, E.; Kaynig, V.; Longair, M.; Pietzsch, T.; Preibisch, S.; Rueden, C.; Saalfeld, S.; Schmid, B., et al. Fiji: an open-source platform for biological-image analysis. Nat Methods 2012, 9, 676-682, doi:10.1038/nmeth.2019. 